DOI: 10.12731/2658-6649-2020-12-3-55-69

УДК 614.2

\title{
БАЛЛЬНО-РЕЙТИНГОВАЯ СИСТЕМА (БРС) ОЦЕНКИ МЕДИЦИНСКОЙ ЭФФЕКТИВНОСТИ ШКОЛЫ ЗДОРОВЬЯ (НА ПРИМЕРЕ ПАЦИЕНТОВ С МНОЖЕСТВЕННОЙ ЛЕКАРСТВЕННОЙ УСТОЙЧИВОСТЬЮ МУСОВАСТЕRIUМ TUBERCULOSIS)
}

\author{
Чубарян В.Т., Саенко Г.И., \\ Худоногов И.Ю., Пустошилова Э.А.
}

Актуальность: исследования эффективности информационно-когнитивных взаимодействий врачей и пациентов в рамках работы школ здоровья (ШЗ) проводятся достаточно часто. Однако они игнорируют клиническую эффективность посещения пациентами занятий ШЗ, особенно, если это пациенты с множественной лекарственной устойчивостью Mycobacterium tuberculosis.

Цель: разработать балльно-рейтинговую систему оценки медицинской эффективности занятий в ШЗ и внедрить её в работу практического здравоохранения.

Методы: клинический (разработка и апробачия на практике объективных шкал для определения исходного уровня тяжести состояния и результативности лечения пациентов с МЛУ/ШЛУ МВТ); лабораторный (выкопировка из историй болезни данных о морфо-функииональных характеристиках пациентов): социологический (выкопировка из историй болезни данных о сочиально-экономическом положении паџиентов с МЛУ/ ШЛУ МВТ); статистический - мы использовали ранговую корреляцию, t-критерий, логическую и линейную регрессии, темп роста относительных величин, угловые коэффичиенты линейных трендов и показатели достоверности аппроксимации для анализа данных о когорте (74 мужчины) пациентов ГБУ РО «ОКЦФП» среднего возраста, из которых 40 занимались в ШЗ, а 34 (группа контроля) - нет.

Результаты: темп роста угловых коэффициентов в формулах линейных регрессий для исследованных групп свидетельствовал о том, что скорость «набора» положительной динамики у пациентов из группы по- 
сещуавиих ШЗ была на 33\% выше, чем у не посещуавиих, также выше на 18,8\% у них была среднегрупповая балльная оценка результативности лечения.

Заключение: внедрение в работу фтизиатрической службы (ФС) объективных БРС оченки исходного состояния и результативности лечения пацциентов с МЛУ/ШЛУ МБТ на групповом уровне позволяют сравнивать медицинскую эффективность аналогичных отделений различных МО или изменения в работе одного отделения за разные промежутки времени и облегчают вхождение ФС в единое информационно-медицинское пространство РФ.

Ключевые слова: пациенты с множественной лекарственной устойчивостью; тусоbacterium tuberculosis; структурирование морфо-функциональных представлений; социокультурная медикализация; информационно-когнитивные воздействия; медицинская эффективность; балльно-рейтинговая система; клинические и лабораторные данные.

\title{
POINT-RATING SYSTEM (PRS) FOR ASSESSING THE MEDICAL EFFICIENCY OF THE HEALTH SCHOOL (ON THE EXAMPLE OF MDR/XDR MBT MALE PATIENTS)
}

\author{
Chubaryan V.T., Saenko G.I., \\ Khudonogov I.Yu., Pustoshilova E.A.
}

Relevance: research on the effectiveness of information and cognitive interactions between doctors and patients in the framework of health schools (HS) is carried out quite often. However, they ignore the clinical effectiveness of attending HS by patients, especially if they are patients with multidrug-resistant Mycobacterium tuberculosis.

Goal: to develop a point-rating system for evaluating the medical effectiveness of classes in HS and implement it in the work of practical health care.

Methods: clinical (development and testing in practice of objective scales for determining the initial level of severity of the condition and the effectiveness of treatment of patients with MDR / XDR MBT); laboratory (copying from medical histories of data on morpho-functional characteristics of patients): sociological (copying from medical histories of data on the socio-economic status of patients with MDR / XDR MBT); statistical - we used rank correla- 
tion, T-criteria, logical and linear regression, growth rate of relative values, angular coefficients of linear trends, and approximation reliability indicators to analyze data on a cohort (74 men) of middle-aged patients of the Regional Clinical Phthisiopulmonology Center, of whom 40 were engaged in HS, and 34 (control group) were not.

Results: the growth rate of angular coefficients in linear regression formulas for the studied groups indicated that the rate of "set" of positive dynamics in patients from the group who visited the HS was 33\% higher than in those who did not attend, and they also had have an average of treatment effectiveness group score by $18.8 \%$ higher.

Conclusion: implementation of the PRS objective estimation of the initial state and efficiency of treatment of patients with MDR/XDR MBT at the group level allow you to compare the medical effectiveness of similar offices in various $M O$ or changes in one Department for different time intervals and ease the association of TB services to a single information and medical space of the Russian Federation.

Keywords: $M D R / X D R$ MBT male patients; structuring of morpho-functional representations; socio-cultural medicalization; information and cognitive effects; medical efficiency; point-rating system; clinical and laboratory data.

\section{Введение}

Медикализация населения - это неизбежный процесс активного распространения медицинского мировоззрения на все сферы общественной жизни [1] посредством проникновения в массовое сознание медицинского дискурса и стиля мышления, медицинских концепций и представлений о причинах болезней, способах их лечения и профилактики. Врачи сегодня не только лечат, но расширяют свое присутствие в информационном пространстве [2, 3]. На платформах СМИ и интернета регулярно проводятся ток-шоу, беседы, лекции о морфо-функциональных особенностях человеческого организма, фармакокинетике, фармакодинамике, новых методах лечения и проч., также все более активно используются информационные площадки, расположенные на территории самих медицинских организаций (MO), открываются школы здоровья (ШЗ) для пациентов различного профиля. Перед посещением таких школ и после окончания цикла обучения пациентам предлагают заполнять специальные анкеты, которые позволяют оценивать объемы полученных в ходе обучения знаний, приверженность к соблюдению рекомендаций врача по своему заболеванию. Для повышения эффективности работы ШЗ внедряются новые образовательные техноло- 
гии, в качестве слушателей привлекаются лица молодого и трудоспособного возраста, расширяются телемедицинские сети, как индивидуальные, так и в организованных коллективах, на предприятиях и в МО. Для закрепления полученных знаний пациентов привлекают к повторному посещению занятий [4]. Оценка эффективности занятий в Ш3 обычно проводится с использованием анкет, которые позволяют выявлять факторы риска определенного заболевания и их влияния на здоровье, интенсивность профилактических мероприятий (повышение физической активности, приверженность к здоровому питанию, изменение отношения к стрессовым ситуациям, отказ от курения и т.д.). При этом было установлено, что ШЗ оказывают больший эффект на женщин, чем на мужчин [5], что может объяснить повышенный интерес именно к пациентам-мужчинам. Другой подход к оценке эффективности работы ШЗ заключается в сравнении полученных индикаторов уровня здоровья с рекомендованными и утвержденными Всероссийскими научными обществами, ассоциациями специалистов и др. [6]. Глобальная профилактическая среда в РФ становится объективной реальностью, которая постоянно расширяется и вовлекает все новые кластеры отечественного здравоохранения. Одним из таких кластеров является Фтизиатрическая служба Российской Федерации, которой приходится сталкиваться с медико-биологическим феноменом множественной лекарственной устойчивости (МЛУ) и широкой лекарственной устойчивости (ШЛУ) Mycobacterium tuberculosis (МБТ) [7]. Указанный феномен синхронизируется с достаточно специфичной социальной средой [8], которая в значительной степени обуславливает возникновение и прогрессирование фтизиатрического процесса. Следовательно, и профилактические мероприятия (занятия в ШЗ), корреспондирующие к межличностным взаимодействиям и трансформирующие микросоциум пациентов должны приводить к заметным изменениям медико-биологических индикаторов.

Вышесказанное определило цель нашего исследования: разработать балльно-рейтинговую систему оценки медицинской эффективности занятий в ШЗ и исходного уровня здоровья пациентов с МЛУ/ШЛУ МБТ и внедрить её в работу практического здравоохранения.

\section{Материал и методы}

В период с апреля по июль 2018 года в исследование было включено

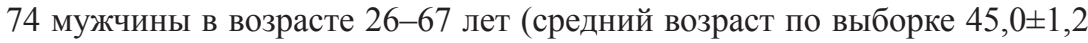
лет, повторно госпитализированных, с хроническим течением туберкулезного процесса в легких. Из них 91,3\% являлись бактериовыделителями, 
8,7\% - МБТ (-), все относились к группе диспансерного учета 2А. Исходное состояние при поступлении (по данным титульного листа истории болезни) расценивалось как удовлетворительное (в 92,9\% случаев) или средней тяжести (в 7,1\%). Единицы наблюдения включались в выборку с учетом следующих критериев: мужской пол (традиционная стандартная химеотерапия оказывает на мужчин меньший эффект, чем на женщин, и они всегда преобладают в специализированных отделениях для пациентов с МЛУ/ШЛУ МБТ); подтвержденный диагноз МЛУ/ШЛУ-туберкулез органов дыхания; подписанное информированное согласие на обработку персональных данных; нахождение на стационарном лечении в Отделении для больных туберкулезом с МЛУ возбудителя №2 ГБУ РО «ОКЦФП».

В течение трех месяцев пациентам предлагалось посещать занятия ШЗ, которые проходили еженедельно в холле стационара и включали демонстрацию презентаций о строении и функциях человеческого организма с фтизиатрическими комментариями.

Для оценки влияния ШЗ на результативность лечения была разработана специальная шкала (табл. 1), которая позволила рассчитать среднегрупповой балл успешности пребывания в стационаре. Итоговая оценка формировалась на основе суммирования баллов всех пациентов с последующим делением на численность группы, что и определяло групповой рейтинг.

Таблица 1.

Экспертная балльно-рейтинговая оценка результата лечения пациентов, учитывающая общее состояние при выписке

\begin{tabular}{|l|c|}
\hline \multicolumn{1}{|c|}{ Результативный признак } & $\begin{array}{c}\text { Оценка результативности } \\
\text { лечения (в баллах) }\end{array}$ \\
\hline Клинико-рентгенологическое улучшение & 5 \\
\hline Клиническое улучшение & 4 \\
\hline $\begin{array}{l}\text { Незначительное клинико-рентгенологическое } \\
\text { улучшение }\end{array}$ & 3 \\
\hline Лабораторное улучшение & 3 \\
\hline Выписан за нарушение режима & 2 \\
\hline Без существенной динамики & 1 \\
\hline Направлен на хирургическое лечение & 1 \\
\hline Необходима интенсивная химиотерапия & \\
\hline
\end{tabular}

Оцифровывание и последующее ранжирование качественных результативных характеристик выписных эпикризов позволили рассчитать кумулятивные среднегрупповые показатели $[9,10]$ и их достоверность, а также 
построить прогностическую модель изменения клинической результативности лечения пациентов с МЛУ/ШЛУ МБТ на фоне участия в занятиях ШЗ. Данные собирались после выписки пациентов из стационара из историй болезней стационарных больных - Ф. № 003, выписки из историй болезней и амбулаторных карт больных туберкулезом - Ф. № 081. Кратность посещений ШЗ сопоставлялась с 68 количественными клиническим и лабораторными признаками (Возраст; Рост; Масса тела; ИМТ; ЧДД; ЧСС; САД; ДАД; Температура тела; биохимические; электрографические; спирометрические параметры).

Статистическая обработка результатов велась по двум основным направлениям:

1) корреляционные связи рассчитывались в масштабах всей выборки и учитывали все кратности посещения Ш3, включая «0»; 2) группировка выборки на участников Ш3 («1+»=1 и более посещений) и неучастников («0» посещений) с последующим определением достоверности групповых различий.

Анализ полученных данных выполнялся с помощью статистического пакета программы Microsoft Office 2016 (Microsoft Excel 2016 MSO версия 16.6.10223.20104). Рассчитывалась ошибка представительности (s) для коэффициента корреляции (КК), относительных и средних величин (m). Для определения достоверности сравниваемых групповых показателей использовался критерий Стьюдента (t). Результаты вычислений, вероятность ошибки которых превышала критическую величину $(\mathrm{p}=0,05)$, рассматривались как подтверждение нулевой гипотезы об отсутствии значимых различий. Для расчета относительной вероятности появления альтернативных признаков было использовано уравнение логической регрессии - odds ratio (OR) - и доверительный интервал - confidence interval (CI) [11]. Группы «1+» и «0» сравнивались также с использованием непараметрической «Mann-Whitney U Test»-системы, предоставленной free on-line калькулятором [12]. Для прогностического моделирования результатов лечения осуществлялось построение линейных трендов, расчет угловых коэффициентов и показателей аппроксимации.

\section{Результаты исследования}

54\% пациентов добровольно посещали занятия ШЗ (от 1 до 6 посещений из 10 занятий, проходивших еженедельно), на основании чего пациенты ретроспективно были разделены на 2 группы - посещавших $(\mathrm{n}=40)$ и не посещавших (n=34) ШЗ.

Корреляционная зависимость для большей части (70,6\%) проанализированных признаков, включая режим терапии, не подтвердилась. 
Несмотря на сходство больных по клиническим формам туберкулеза и основному критерию - МЛУ/ШЛУ МБТ, разброс некоторых показателей, тем не менее, оказался значимым с точки зрения статистики. В таблице 2 приведены признаки со связью средней силы с кратностью посещений ШЗ. Все указанные признаки отражали состояние пациентов на момент выписки. Для удобства анализа исследуемые признаки были разделены на две группы: электрокардиографические и прочие.

Таблииа 2.

Данные по всей выборке о значимых корреляционных связях между кратностью посещений ШЗ пациентами с МЛУ/ШЛУ МБТ и некоторыми клинико-лабораторными данными, которые были выкопированы после выписки $(\mathbf{p}<0,05)$

\begin{tabular}{|c|c|c|}
\hline Параметр & KK (r) & $\mathrm{p}$ \\
\hline \multicolumn{3}{|l|}{ Электрокардиографически опосредованные связи } \\
\hline Угол альфа отклонения электрической оси желудочков (в градусах) & $-0,461$ & $<0,05$ \\
\hline Угол альфа отклонения электрической оси сердца (в градусах) & $-0,408$ & $<0,05$ \\
\hline Длительность зубца Р (в мс) & $-0,329$ & $<0,05$ \\
\hline \multicolumn{3}{|l|}{ Прочие связи } \\
\hline Глюкоза в сыворотке крови (в ммоль/литр) & $-0,460$ & $<0,05$ \\
\hline Систолическое артериальное давление (САД) (мм рт.ст.) & 0,303 & $<0,05$ \\
\hline
\end{tabular}

Как следует из табл. 3, у больных мужчин с МЛУ/ШЛУ МБТ рост приверженности к посещениям занятий ШЗ и изменения гемодинамических и метаболических параметров значимо взаимосвязаны.

Группировка выборки по качественному критерию посещаемости/непосещаемости ШЗ при допущении о существовании нормального распределения количественных признаков позволила выявить следующие значимые различия

Таблица 3.

Данные по всей выборке о значимых корреляционных связях между кратностью посещений ШЗ пациентами с МЛУ/ШЛУ МБТ и некоторыми клинико-лабораторными данными, которые были выкопированы после выписки, а получены и записаны лечащим врачом перед выпиской $(\mathbf{p}<0,05)$

\begin{tabular}{|l|c|c|c|c|c|}
\hline \multirow{2}{*}{} & \multicolumn{2}{|c|}{$« 0 »$} & \multicolumn{2}{|c|}{$« 1+»$} & \multirow{2}{*}{$\mathrm{t}_{\mathrm{M}}$} \\
\cline { 2 - 6 } & $\mathrm{M}$ & $\pm \mathrm{m}$ & $\mathrm{M}$ & $\pm \mathrm{m}$ & \\
\hline САД (мм рт. ст.) & 114,1 & 0,9 & 117,5 & 1,1 & 2,4 \\
\hline Моноциты (в\%) & 6,1 & 0,2 & 6,8 & 0,3 & 2,0 \\
\hline
\end{tabular}


Окончание табл. 3.

\begin{tabular}{|l|c|c|c|c|c|}
\hline Креатинин (мкмоль/л) & 120,8 & 16,3 & 82,9 & 2,7 & 2,3 \\
\hline Мочевина (ммоль/л) & 7,3 & 0,6 & 5,1 & 0,3 & 3,3 \\
\hline Длительность зубца Р (в мс) & 113,1 & 1,4 & 100,6 & 1,5 & 6,0 \\
\hline Длительность интервала QТс (в мс) & 378,9 & 2,2 & 368,6 & 2,0 & 3,5 \\
\hline $\begin{array}{l}\text { Угол альфа отклонения электрической оси } \\
\text { предсердий (в градусах) }\end{array}$ & 80,2 & 1,5 & 74,7 & 1,5 & 2,6 \\
\hline $\begin{array}{l}\text { Угол альфа отклонения электрической оси } \\
\text { желудочков (в градусах) }\end{array}$ & 75,7 & 2,5 & 67,2 & 1,5 & 2,9 \\
\hline ЧСС & 93,0 & 2,4 & 84,0 & 2,2 & 2,7 \\
\hline Индекс Тифно & 29,5 & 0,0 & 55,9 & 3,3 & 8,1 \\
\hline Индекс Генслара & 100,0 & 0,0 & 72,1 & 5,1 & 5,5 \\
\hline Прослушал лекций в часах & 0,0 & 0,0 & 2,4 & 0,2 & 10,2 \\
\hline
\end{tabular}

Проверка выявленных различий осуществлялась при помощи теста Манна-Уитни, который позволил сделать несколько уточнений и дополнений, также опровергающих нулевую гипотезу о тождественности групп (табл. 4).

\section{Значимые различия количественных признаков в группах пациентов}

Таблицча 4.

с МЛУ/ШЛУ МБТ, посещавших $(n=40)$ и не посещавших $(n=34)$ Ш3

( $\mathrm{Z}$ и U тесты по Манну-Уитни, находящиеся вне приемлемого диапазона, подтверждающего тождество выборок с вероятностью 95\%)

\begin{tabular}{|l|c|c|c|c|}
\hline \multicolumn{1}{|c|}{ Сравниваемый признак } & Z-тест & $\begin{array}{c}\text { Приемлемый } \\
\text { диапазон }\end{array}$ & U-тест & $\begin{array}{c}\text { Приемлемый } \\
\text { диапазон }\end{array}$ \\
\hline $\begin{array}{l}\text { Систолическое артериаль- } \\
\text { ное давление (САД) }\end{array}$ & 2.209814 & $-1.9600: 1.9600$ & 833.00 & $480.1400: 811.8600$ \\
\hline $\begin{array}{l}\text { Диастолическое артери- } \\
\text { альное давление (ДАД) }\end{array}$ & 2.088562 & $-1.9600: 1.9600$ & 820.00 & $482.7100: 809.2900$ \\
\hline Мочевина в сыворотке крови & -2.852992 & $-1.9600: 1.9600$ & 355.00 & $429.1800: 754.8200$ \\
\hline Длительность интервала P & -4.036376 & $-1.9600: 1.9600$ & 74.00 & $164.5400: 335.4600$ \\
\hline Длительность интервала QТс & -2.518890 & $-1.9600: 1.9600$ & 140.00 & $164.4100: 335.5900$ \\
\hline $\begin{array}{l}\text { Угол альфа отклонения элек- } \\
\text { трической оси предсердий }\end{array}$ & -2.544993 & $-1.9600: 1.9600$ & 139.00 & $164.5200: 335.4800$ \\
\hline $\begin{array}{l}\text { Угол альфа отклонения элек- } \\
\text { трической оси желудочков }\end{array}$ & -1.881209 & $-1.9600: 1.9600$ & 168.00 & $164.5700: 335.4300$ \\
\hline $\begin{array}{l}\text { Частота сердечных сокра- } \\
\text { щений (ЧСС) }\end{array}-2.058799$ & $-1.9600: 1.9600$ & 160.00 & $164.3200: 335.6800$ \\
\hline
\end{tabular}

Так, группа пациентов, не посещавших занятия ШЗ вообще, значимо превышала соответствующие показатели группы посещавших занятия по следующим критериям: «Мочевина в сыворотке крови», «Длительность интервала 
Р», «Длительность интервала QТс», «Угол альфа отклонения электрической оси предсердий», «Угол альфа отклонения электрической оси желудочков», «ЧСС»; имела достоверно более низкие значения по таким показателям, как «САД», «ДАД». Вместе с тем, на выходе (после выписки из стационара) более высокого клинического результата достигла группа «1+» (принимавшая участие в работе ШЗ). При этом необходимо отметить, что по отношению ко всей выборке применялась терапия, соответствовавшая стандартным режимам, которая практически не менялось в течение всего исследования (табл. 5).

Таблица 5.

Среднегрупповая результативность лечения пациентов с МЛУ/ШЛУ МБТ посещавших и не посещавших ШЗ (в [\%*балл])

\begin{tabular}{|c|c|c|c|c|c|}
\hline \multirow{2}{*}{$\begin{array}{c}\text { X - оценка } \\
\text { результатив- } \\
\text { ности } \\
\text { лечения } \\
\text { (в баллах) }\end{array}$} & \multirow[b]{2}{*}{ Результативный признак } & \multicolumn{2}{|c|}{$\begin{array}{c}\text { Не посещали } \\
\text { Ш3 }(\mathrm{n}=34) \\
\end{array}$} & \multicolumn{2}{|c|}{$\begin{array}{l}\text { Посещали } \\
\text { Ш3 (n=40) }\end{array}$} \\
\hline & & $\begin{array}{c}\text { Удель- } \\
\text { ный вес } \\
\text { признака } \\
(\mathrm{P} * \mathrm{X})\end{array}$ & $\pm \mathrm{m}$ & $\begin{array}{c}\text { Удель- } \\
\text { ный вес } \\
\text { признака } \\
(\mathrm{P} * \mathrm{X})\end{array}$ & $\pm \mathrm{m}$ \\
\hline 5 & $\begin{array}{l}\text { Клинико-рентгенологическое } \\
\text { улучшение }\end{array}$ & 29,4 & 4,0 & 37,5 & 4,2 \\
\hline 4 & Клиническое улучшение & 70,6 & 6,5 & 60,0 & 5,6 \\
\hline 3 & $\begin{array}{l}\text { Незначительное клинико-рентге- } \\
\text { нологическое улучшение }\end{array}$ & 0,0 & 0,0 & 15,0 & 3,4 \\
\hline 3 & Лабораторное улучшение & 0,0 & 0,0 & 15,0 & 3,4 \\
\hline 3 & Выписан за нарушение режима & 35,3 & 5,5 & 45,0 & 5,6 \\
\hline 2 & Без существенной динамики & 70,6 & 8,2 & 100,0 & 7,9 \\
\hline 1 & $\begin{array}{l}\text { Направлен на хирургическое } \\
\text { лечение }\end{array}$ & 11,8 & 5,5 & 0,0 & 0,0 \\
\hline 1 & $\begin{array}{l}\text { Необходима интенсивная хими- } \\
\text { отерапия }\end{array}$ & 11,8 & 5,5 & 0,0 & 0,0 \\
\hline & Всего & 229,5 & 14,8 & 272,5 & 12,9 \\
\hline
\end{tabular}

Разность среднегрупповой балльной оценки результативности (БОР) лечения оказалась достоверна $(\mathrm{t}=2,2)$ - показатели БОР группы посещавшей лекции на 18,8\% превышал соответствующий показатель в контрольной группе. Графическое отображение данных таблицы 5, представленное на рисунке, позволило сравнить угловые коэффициенты [13] в формулах линейных регрессий для исследованных групп и установить, что скорость «набора» положительной динамики у пациентов из группы посещавших Ш3 на 33\% выше. 


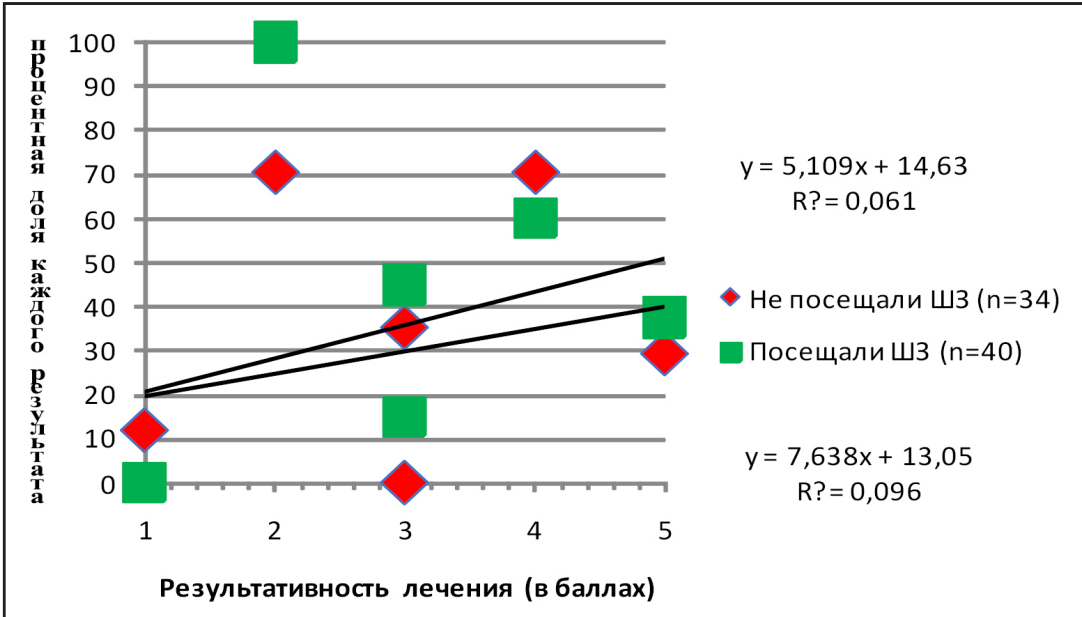

Рис. Результативность лечения в группах пациентов с МЛУ/ШЛУ МБТ, посещавших $(n=40)$ и не посещавших $(n=34)$ Ш3

Не менее важным аспектом нашего исследования явилось определение медико-социальных предикторов возникновения МЛУ/ШЛУ МБТ, чувствительных к медико-информационным воздействиям (занятиям в ШЗ). Рассматривая вопрос о позитивном отношении пациентов к занятиям в ШЗ (табл. 6), мы сконцентрировались на одной из наиболее важных составляющих жизнедеятельности пациентов - на их трудовой активности [14], которая была представлена в историях болезни посредством двух альтернативных индикаторов включенности в профессиональный социум: «Не работаю» и «Владею профессией ....».

Таблийа 6.

Значимые различия индикаторов включенности

в профессиональный социум в группах пациентов с МЛУ/ШЛУ МБТ, посещавших $(n=40)$ и не посещавших Ш3 $(n=34)$

\begin{tabular}{|l|c|c|}
\hline \multicolumn{1}{|c|}{ Сравниваемый признак } & OR & CI \\
\hline Не работает & 0.02 & $(0.00,0.09)$ \\
\hline Имеет профессию & 16.0 & $(3.37,75.93)$ \\
\hline
\end{tabular}

По нашим данным они распределились зеркально и значимо. Кроме того, несомненным предиктором активного участия пациентов в занятиях ШЗ мы считаем образование, которое непосредственно связано с будущей профессией. Тем не менее, в паре признаков «Прослушал лекций в ча- 
сах» и «Образование» корреляционный метод позволил выявить незначимую положительную зависимость слабой силы $(\mathrm{r}=0,256: \mathrm{m}=0,130 ; \mathrm{t}=7,671$ : $\mathrm{r} / \mathrm{m}=51 \%$ ), в то время как $\mathrm{Z}$ и $\mathrm{U}$ тесты по Манну-Уитни подтвердили идентичность их распределений ( $\mathrm{Z}=-1.276777$, находится в допустимом диапазоне критических значений 95\%: [-1.9600: 1.9600 ]; $\mathrm{U}=585.50$, находится в приемлемом диапазоне 95\%: [ 524.2300: 875.7700 ]).

\section{Заключение}

Внедрение в работу ФС объективных БРС оценки исходного состояния и результативности лечения пациентов с МЛУ/ШЛУ МБТ на групповом уровне позволяют сравнивать медицинскую эффективность аналогичных отделений различных МО или изменения в работе одного отделения за разные промежутки времени и облегчают вхождение ФС в единое информационно-медицинское пространство РФ.

Всесторонний компаративный анализ клинических и лабораторных данных показал, что процесс организации и проведения занятий ШЗ для пациентов с МЛУ/ШЛУ МБТ не только расширяет круг их общения и восполняет дефицит коммуникаций, но также связан с течением основного заболевания, его осложнений и сопутствующей патологии.

В ходе исследования были установлены медико-социальные предикторы, значимо повышающие медицинскую эффективность работы ШЗ для пациентов-мужчин. К ним относилось повышение уровня образования и активное участие в профессиональной деятельности.

\section{Список литературы}

1. Данилевская Т.В., Элланский Ю.Г., Худоногов И.Ю. Количественный анализ значимости источников медикализации в социокультурной среде мегаполиса // Социология медицины. 2017;16.(2):94-99.

2. Худоногов И.Ю., Иванов А.С., Зарубинская Л.Г. и др. Научное обоснование когнитивно-информационного протезирования (медико-социологический анализ естественной репарации самосохранительных мотивационных матриц) // В мире научных открытий. 2018;10(3):41-61.

3. Худоногов И.Ю., Липилкин П.В., Ким В.В. Информационно-когнитивная эффективность деятельности врача как индикатор качества медицинской помощи // В мире научных открытий. 2017;9(2):200-213.

4. Беляева А.К., Мультановский Б.Л., Шабардина С.В. и др. Эффективность работы школы здоровья для пациентов с артериальной гипертонией // Здоровье, демография, экология финно-угорских народов. 2013;4:29-31. 
5. Орлова Е.В., Тишкина Н.В. Оценка влияния школы здоровья на модифицируемые факторы риска артериальной гипертонии. Вестник новых медицинских технологий. 2010;17(2):293-295.

6. Чазова И.Е., Ощепкова Е.В. Итоги реализации федеральной целевой программы по профилактике и лечению артериальной гипертензии в России в 20022012 гг. // Вестник Российской академии медицинских наук. 2013;68(2):4-11.

7. Борзенко А.С., Калуженина А.А., Шмелёв Э.Н. и др. Новые режимы в терапии туберкулеза с множественной лекарственной устойчивостью // Аллергология и иммунология. 2013;14(1):45.

8. Мижидон А.Д., Рыгзынова М.В. Построение математической модели влияния экономических факторов на динамику социально значимых заболеваний // Вестник Бурятского государственного университета. 2012;S4:87-91.

9. Илюхина О.В., Элланский Ю.Г., Худоногов И.Ю. Методические вопросы применения структурированных индикаторов в медико-социологических исследованиях // Социология медицины. 2013;1(22):15-18.

10. Худоногов И.Ю., Осадчук О.А. Методология оценки достоверности ранжированных индикаторов медико-социальных процессов // Обмен веществ при адаптации и повреждении (дни молекулярной медицины на Дону): Материалы XIII Российской научно-практической конференции с международным участием. 2014. С. 193-196.

11. Select Statistical Services Ltd. Oxygen House, Grenadier Road, Exeter Business Park, Exeter, UK, «On-line Calculators» [Электронный pecypc]. URL: https:// select-statistics.co.uk/calculators/ (дата обращения: 12.08.2019).

12. Statistics Kingdom, statskingdom@gmail.com, Mann Whitney U Test. [Электронный ресурc]. URL: http://www.statskingdom.com/170median_mann_ whitney.html (дата обращения: 12.08.2019).

13. Худоногов И.Ю. Социальное регулирование самосохранительного поведения // Общественное здоровье как функция информационно-когнитивного пространства. Beau Bassin, Mauritius: LAP LAMBERT; 2018.

14. Худоногов И.Ю. Индикаторная модель взаимодействия общественного здоровья и социально-профессиональной среды // Современные подходы к продвижению здоровья: Материалы V Международной научно-практической конференции. Редакционный совет: А.Н. Лызиков, Т.М. Шаршакова, И.А. Чешик и др. 2014. С. 268-271.

\section{References}

1. Danilevskaia T.V., Ellansky Yu.G., Khudonogov I.Yu. The quantitative analysis of significance of sources of medicalization in sociocultural environment of 
megalopolis. Sotsiologiya meditsiny [Sociology of Medicine]. 2017;16(2):94-99 (in Russ.).

2. Khudonogov Igor Yurievich, Ivanov Alexander Sergeevich, Zarubinskaya Lyubov Grigoryevna. Scientific substantiation of cognitive-informational prosthetics (medical-sociological analysis of natural reparation of self-preservation motivation matrix). Siberian Journal of Life Sciences and Agriculture. 2018;10(3):41-61 (in Russ.).

3. Khudonogov Igor Yurievich, Lipilkin Pavel Viktorovich, Kim Vyacheslav Vladislavovich. Information-cognitive effectiveness of physician as an indicator of health care quality. Siberian Journal of Life Sciences and Agriculture. 2017;9(2):200-213 (in Russ.).

4. Belyayeva A.K., Multanovskiy B.L., Shabardina S.V. et al. Effectiveness of schools of health for the patients with arterial hypertension. Zdorov'e, demografiya, ekologiya finno-ugorskikh narodov [Health, Demography, Ecology of Finno-Ugric People]. 2013;4:29-31 (in Russ.).

5. Orlova E.V., Tishkina N.V. The evaluation of influence of the health school on modified risk factors of arterial hypertension. Vestnik novykh meditsinskikh tekhnologiy [Journal of New Medical Technologies]. 2010;17(2):293-295 (in Russ.).

6. Chazova I.E., Oschepkova E.V. Results of the Federal (National) Project for prevention and treatment essential hypertension patients in Russia from 2002-2012 years. Vestnik Rossiyskoy akademii meditsinskikh nauk [Annals of the Russian academy of medical sciences]. 2013;68(2):4-11 (in Russ.).

7. Borzenko A.S., Kaluzhenina A.A, Shmelev E.N. et al. New regimes of treatment multi-drug resistant tuberculosis. Allergologiya i immunologiya [Allergology and Immunology]. 2013;14(1):45 (in Russ.).

8. Mizhidon Arsalan Dugarovich, Rygzynova Marina Victorovna. Construction of mathematical model of economic factors influence on dynamics of socially significant diseases. Vestnik Buryatskogo gosudarstvennogo universiteta [The Buryat State University Bulletin]. 2012;S4:87-91 (in Russ.).

9. Ilyukhina O.V., Ellanskiy Yu.G., Khudonogov I.Yu. The technique issues of application of structured indicators in medical sociologic studies. Sotsiologiya meditsiny [Sociology of Medicine]. 2013;1(22):15-18 (in Russ.).

10. Khudonogov I.Ju., Osadchuk O.A. Metodologija ocenki dostovernosti ranzhirovannyh indikatorov mediko-social'nyh processov [Methodology for assessing the reliability of ranked indicators of medical and social processes]. Obmen veshhestv pri adaptacii i povrezhdenii (dni molekuljarnoj mediciny na Donu) Materialy XIII Rossijskoj nauchno-prakticheskoj konferencii s mezhdunarodnym uchastiem [Metabolism during adaptation and damage (days of molecular 
medicine in the Don): Materials of the XIII Russian Scientific and Practical Conference with international participation]. 2014, pp. 193-196.

11. Select Statistical Services Ltd. Oxygen House, Grenadier Road, Exeter Business Park, Exeter, UK, «On-line Calculators». https://select-statistics.co.uk/calculators/ (access date: 12.08.2019).

12. Statistics Kingdom, statskingdom@gmail.com, Mann Whitney U Test. http:/www. statskingdom.com/170median_mann_whitney.html (access date: 12.08.2019).

13. Khudonogov I.Yu. Social regulation of self-preserving behavior. Obshchestvennoe zdorov'e kak funktsiya informatsionno-kognitivnogo prostranstva [Public health as a function of the information and cognitive space]. Beau Bassin, Mauritius: LAP LAMBERT; 2018. (in Russ.).

14. Khudonogov I.Ju. Sovremennye podhody k prodvizheniju zdorov'ja Materialy $V$ Mezhdunarodnoj nauchno-prakticheskoj konferencii [Sovremennye podkhody k prodvizheniyu zdorov'ya: Materialy V Mezhdunarodnoy nauchno-prakticheskoy konferentsii]. Editorial Council: A.N. Lyzikov, T.M. Sharshakova, I.A. Cheshik et al. 2014, pp. 268-271.

\section{ДАННЫЕ ОБ АВТОРАХ}

Чубарян Вартан Тарасович, доктор медицинских наук, профессор, заведующий кафедрой фтизиатрии и пульмонологии ФПК и ППС ФГБОУ ВО РостГМУ Минздрава России

пер. Нахичеванский, 29, г. Ростов-на-Дону, 344022, Российская Федераичя chubar88@yandex.ru

Саенко Галина Ивановна, доктор медицинских наук, врач-фтизиатр высшей категории, главный врач ГБУ РО «ОКЦФП» ул. Орская, 24, г. Ростов-на-Дону, 344065, Российская Федерациия stb3@mail.ru

Худоногов Игорь Юрьевич, кандидат медицинских наук, старший преподаватель кафедры общественного здоровья и здравоохранения №1 с курсом истории медицины ФГБОУ ВО РостГМУ Минздрава России пер. Нахичеванский, 29, г. Ростов-на-Дону, 344022, Российская Федераичя fux1@ya.ru 
Пустошилова Эвелина Андреевна, студент педиатрического факультета ФГБОУ ВО РостГМУ Минздрава России пер. Нахичеванский, 29, г. Ростов-на-Дону, 344022, Российская Федерачия eva.pust84@gmail.com

\section{DATA ABOUT THE AUTHORS}

Chubaryan Vartan Tarasovich, Doctor of Medical Sciences, Professor, Head of the Department of Phthisiology and Pulmonology Rostov State Medical University of the Ministry of Health of Russia 29, Nakhichevansky Str., Rostov-on-Don, 344022, Russian Federation chubar88@yandex.ru

Sayenko Galina Ivanovna, MD, Phthisiatrist of the Highest Category, Head Physician

Regional Clinical Center of Phthisiopulmonology

24, Orskaya Str., Rostov-on-Don, 344065, Russian Federation stb3@mail.ru

Khudonogov Igor Yuryevich, Candidate of Medical Sciences, Senior Lecturer at the Department of Public Health and Healthcare №1 with a History of Medicine Rostov State Medical University of the Ministry of Health of Russia 29, Nakhichevansky Str., Rostov-on-Don, 344022, Russian Federation fux1@ya.ru SPIN-code: 8063-0786 ORCID ID: 0000-0003-1879-012X ResearcherID: B-4399-2016

Pustoshilova Evelina Andreevna, Student of the Pediatric Faculty Rostov State Medical University of the Ministry of Health of Russia 29, Nakhichevansky Str., Rostov-on-Don, 344022, Russian Federation eva.pust84@gmail.com 